\title{
Arrested Pneumatization in Pediatric Age Group: Computed Tomography and Magnetic Resonance Imaging Findings
}

\author{
N. Kemal Altinbas ${ }^{1}$ Gokhan Gural ${ }^{1} \quad$ Altan Gunes $^{1}$ Betul E. Derinkuyu ${ }^{1}$ Havva Akmaz Unlu ${ }^{1}$ \\ ${ }^{1}$ Department of Radiology, Ankara Children's Hematology Oncology \\ Address for correspondence N. Kemal Altinbas, MD, Department of \\ Radiology, Ankara Children's Hematology Oncology Training and \\ Research Hospital, Ankara, Turkey (e-mail: namikaltin@gmail.com).
}

J Pediatr Neuroradiol 2015;4:7-10.

\begin{abstract}
Keywords

- arrested pneumatization

- developmental anomaly

- paranasal sinuses

- skull base

Objective Arrested pneumatization of the skull base is a developmental anatomical variant of the paranasal sinuses. Although it is an incidental finding, severe bone lesions can be confusing in the differential diagnosis. In this study, arrested pneumatization in the pediatric group is discussed with computed tomography (CT) and magnetic resonance imaging (MRI) findings.

Methods Cranial scanning findings of 550 patients who underwent imaging in the Radiology Department of our hospital with various diagnoses between 2012 and 2013 were examined. CT and MRI findings of arrested pneumatization in six patients were evaluated and recorded.

Results The patients ranged in age from 7 to 14 years. The indications for scanning included neurofibromatosis type $1(n=1)$, papilledema $(n=2)$, epilepsy $(n=1)$, sixth nerve palsy $(n=1)$, and headache $(n=1)$. Arrested pneumatization was detected incidentally in all patients. Four patients had arrested pneumatization of the sphenoid bone, and two had arrested pneumatization of the temporal bone (petrous apex).

Conclusion Arrested pneumatization of the skull base is an incidental finding most commonly detected in association with the sphenoid bone. It is a benign imaging finding that can be observed in the pediatric group and may cause diagnostic confusion. Therefore, CT and MRI findings are of great importance for the final diagnosis.
\end{abstract}

\section{Introduction}

Pneumatization of the skull base and paranasal sinuses begins during the intrauterine period and continues until young adulthood. ${ }^{1}$ Ordinarily, the red bone marrow is replaced by fatty bone marrow prior to pneumatization of the paranasal sinuses. Invasion of epithelial cells forming the respiratory mucosa starts after this transformation. If failure at any of the steps defined above occurs, then pneumatization is either decreased or lost. However, the definite mechanism is mostly uncertain. ${ }^{2}$ Individuals exhibiting varied degrees of failure of pneumatization before the respiratory mucosa has fully extended into sites of early fatty marrow conversion are

received

February 24, 2015

accepted after revision

June 7, 2015

published online

September 30, 2015

left with persistent atypical fatty marrow adjacent to the sinus during adulthood. If the variation is not recognized, imaging features may sometimes create diagnostic difficulties or misdiagnoses in the interpretation of skull base computerized tomography (CT) and magnetic resonance imaging (MRI). ${ }^{3}$

Although arrested pneumatization is an incidental finding, serious bone lesions should be kept in mind in the differential diagnosis and this may lead to confusion. Arrested pneumatization has been presented in recent studies, but the current study is the first to describe the issue in the pediatric age group with $\mathrm{CT}$ and MRI findings.

Copyright $\odot 2015$ by Georg Thieme Verlag KG, Stuttgart・ New York
DOI http://dx.doi.org/ $10.1055 / \mathrm{s}-0035-1564665$. ISSN $1309-6680$. 
8 Arrested Pneumatization in Pediatric Age Group Altinbas et al.

Table 1 Sites of involvement and CT and MRI findings of arrested skull base pneumatization.

\begin{tabular}{|l|l|l|}
\hline \multirow{5}{*}{ Sites of involvement $(n=6)$} & & Frequency \\
\hline \multirow{5}{*}{ CT findings $(n=1)$} & Basisphenoid bone & $4 / 6$ \\
\cline { 2 - 3 } & Pterygoid processes & $4 / 6$ \\
\cline { 2 - 3 } & Multiple contiguous sites & $4 / 6$ \\
\cline { 2 - 3 } & Petrous temporal apex & $2 / 6$ \\
\hline \multirow{5}{*}{ MR findings $(n=6)$} & Well-circumscribed, sclerotic margins, with narrow transition zone & $1 / 1$ \\
\cline { 2 - 3 } & Regions of internal fat density zones & $1 / 1$ \\
\cline { 2 - 3 } & Regions of internal soft-tissue density & $1 / 1$ \\
\cline { 2 - 3 } & Curvilinear internal calcifications & $3 / 6$ \\
\hline & Regions of increased T1 signal (fat signal) & $6 / 6$ \\
\cline { 2 - 3 } & Predominantly high signal on spin-echo T2-weighted images & $0 / 6$ \\
\cline { 2 - 3 } & Predominantly low signal on spin-echo T2-weighted images \\
\cline { 2 - 3 } & Postgadolinium contrast enhancement $(n=2)$ & \\
\hline
\end{tabular}

\section{Materials and Methods}

Among 550 patients who were admitted for cranial examination to our Hospital's Radiology Department during 2012 and 2013 for various disease entities, CT and MRI findings of six patients exhibiting arrested pneumatization were evaluated retrospectively. Approval from the local ethics committee was obtained for the study.

Images were assessed using the imaging criteria previously described by Welker et al. ${ }^{3}$ Patients with other confounding pathologies, such as osteomyelitis, ossifying fibroma, multiple myeloma, chondrosarcoma, chordoma, or bone metastases, were excluded.

The affected regions of the bone defined via CT and MR findings were noted ( - Table 1). Age, symptoms, gender of the patients, and imaging indications were recorded.

CT examinations were performed with a Siemens CT scanner (SOMATOM Spirit). CT images were obtained with a helical scanner in accordance with clinical requirements in the cranial areas with slice thicknesses of 1 to $5 \mathrm{~mm}$, and axial or coronal planes without the use of contrast media.

All MR examinations were performed with 1.5 Tesla MR scanner (Infinion; Philips, Cleveland, Ohio, United States) using 5-mm slice thickness. The diagnosis was verified with the use of contrast media in patients with type 1 neurofibromatosis (NF) and in the patient examined for sixth cranial nerve paralysis. MR venography was additionally performed for two patients in seeking for the cause of papilledema.

\section{Results}

The study group had an age distribution of 7 to 14 years old. The distribution of the patients with arrested pneumatization was as follows: one with a diagnosis of NF type $1(n=1)$, two with the etiological diagnosis of papilledema $(n=2)$, one with epilepsy assessment $(n=1)$, one for an examination of the sixth nerve paralysis $(n=1)$, and one for a headache etiology ( $n=1)$. The variation was incidentally identified in these patients while searching for the reasons mentioned above. Arrested pneumatization was observed in four patients in the sphenoid bone (-Fig. 1) and in two patients in the temporal bone ( $\mathbf{- F i g . 2}$ ). Only one patient had both $\mathrm{CT}$ and MR scans, while the other five patients received MRI. MR venography was performed in two patients seeking for the cause of papilledema.

When evaluated according to the sites of bone involvement, the basisphenoid bone in four cases, the pterygoid process in four cases, and multiple adjacent regions in four cases were identified. Neither clivus nor occiput involvement was found in the study group. Different from other studies in the literature, two of our patients had temporal bone apex involvement (-Figs. 2 and $\mathbf{3}$ ) (-Table $\mathbf{1}$ ).

The patient who received a CT scan had findings of wellcircumscribed, sclerotic margins, with narrow transition zone,

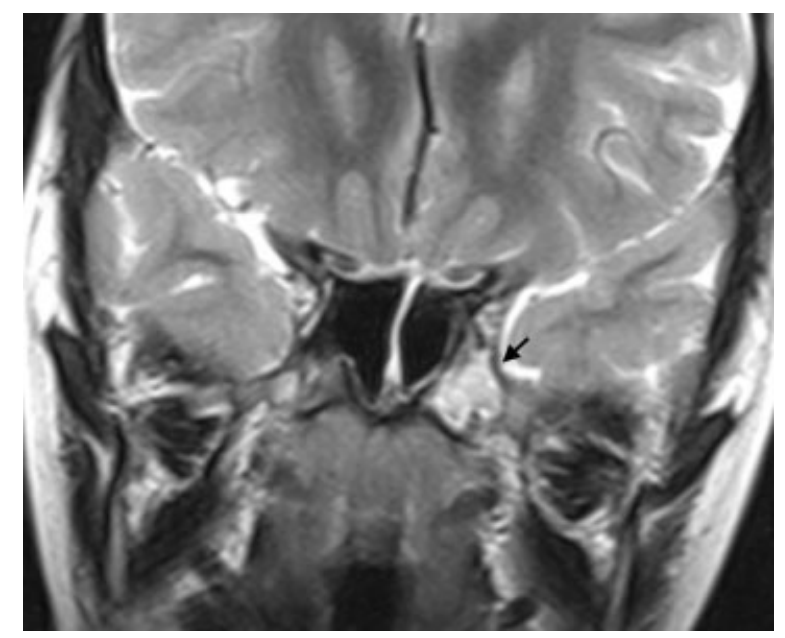

Fig. 1 On T2-weighted image, arrested pneumatization exhibiting a characteristic hyperintense signal on the left side of the sphenoid bone (black arrow). 


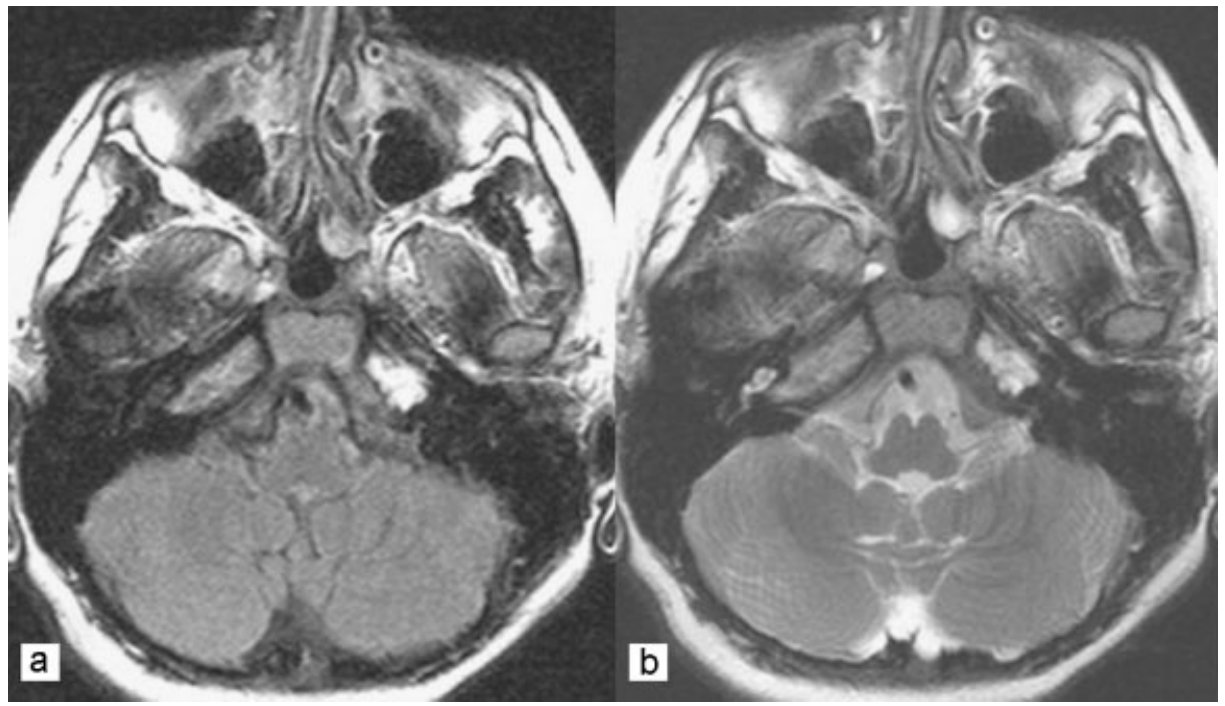

Fig. 2 Hyperintense signal characteristic of arrested pneumatization visible at the left petrous temporal apex on T1- (a) and T2-weighted images (b).
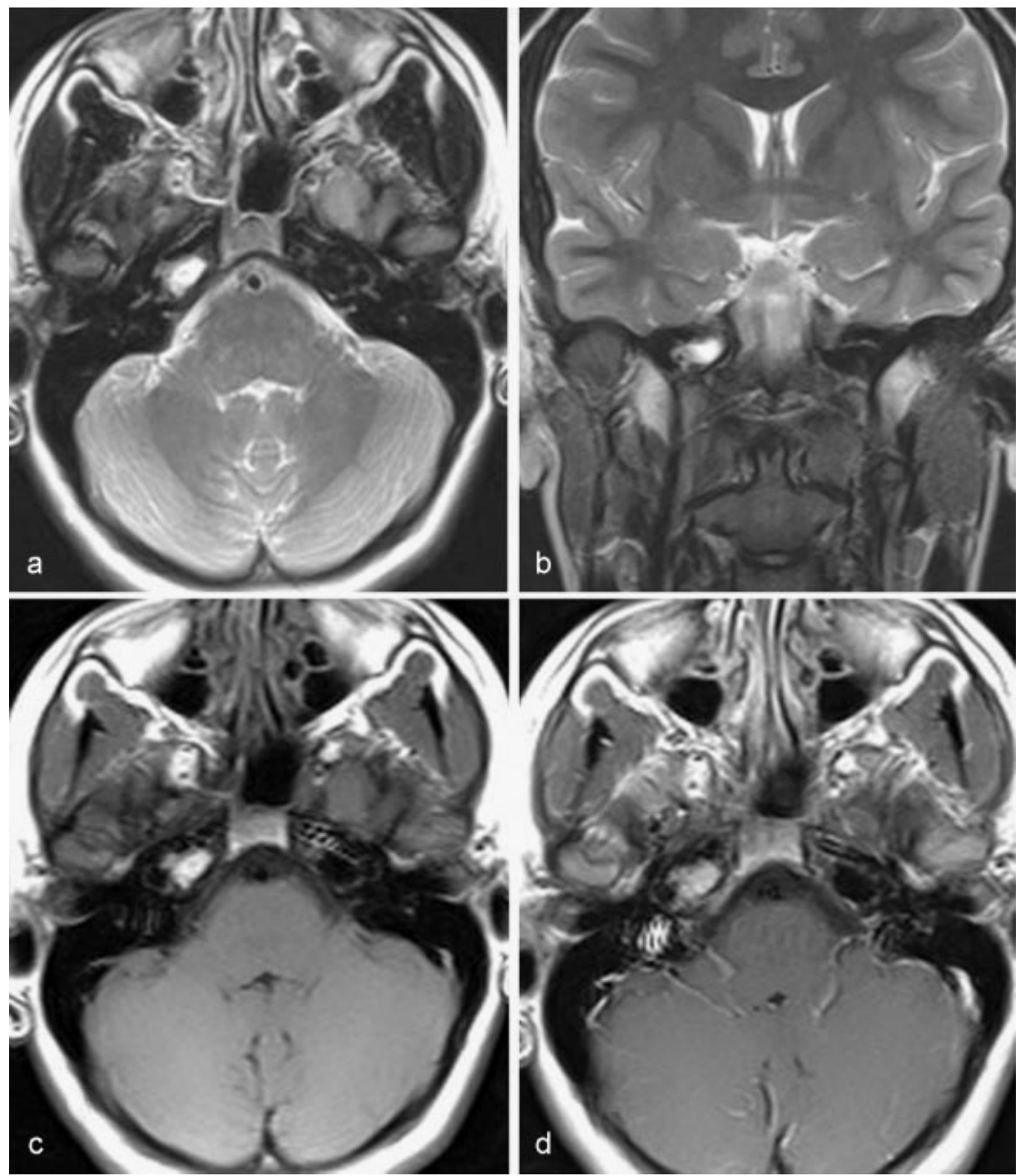

Fig. 3 On MR sections: hyperintense signal at the right temporal petrous apex on axial and coronal T2-weighted image (a, b), on T1-weighted image, hyperintense signal characteristic of fat content (c), no enhancement after gadolinium injection (d) indicative of arrested pneumatization. 
regions of internal fat density zones, internal soft-tissue density, and curvilinear internal calcifications (-Fig. 4) (-Table 1).

When evaluated according to MRI findings, three of six patients had an increased $\mathrm{T} 1$ signal region (fatty). Six patients had a predominantly high signal on T2-weighted images. None of the patients exhibited low signal predominance on T2-weighted images. For two patients who received a gadolinium injection, no enhancement was observed (-Table 1). Two patients who received MR venography did not exhibit any anomalies.

\section{Discussion}

Arrested pneumatization is most frequently observed in relation to sphenoid sinus and is a developmental anomaly observable in normal and/or known accessory pneumatization areas. It generally manifests itself in the basisphenoid bone and pterygoid process region, ${ }^{3,4}$ with the petrous temporal apex and clivus also being reported areas. ${ }^{5,6}$ In fact, accessory pneumatization areas such as the occipital and frontal bones, as well as the maxillary bone, have also been defined. ${ }^{3,4}$ However, in the ethmoid bone, arrested pneumatization has not been defined, likely due to its aeration mechanism. ${ }^{4}$ In our cases, lesions were defined in the sphenoid bone and sphenoid subsites (-Fig. 1), as well as in the temporal bone petrous apices. The temporal bone petrous apex has been defined only in one previous case report, ${ }^{5}$ with a lesion involving the sphenoid bone, pterygoid process and petrous apex. Two of our cases differed from other cases in the literature as far as the involvement of only the petrous apex is concerned (-Fig. 2).

Arrested pneumatization has generally been defined as an incidental imaging finding., ${ }^{3,6}$ De Jong et al reported in a case presentation that for several years their patient suffered headache, photophobia, intermittent nausea, and vomiting, but no characteristic feature was identified from physical, neurologic, and laboratory findings. ${ }^{5}$ In our cases also, arrested pneumatization was incidentally identified at diag-

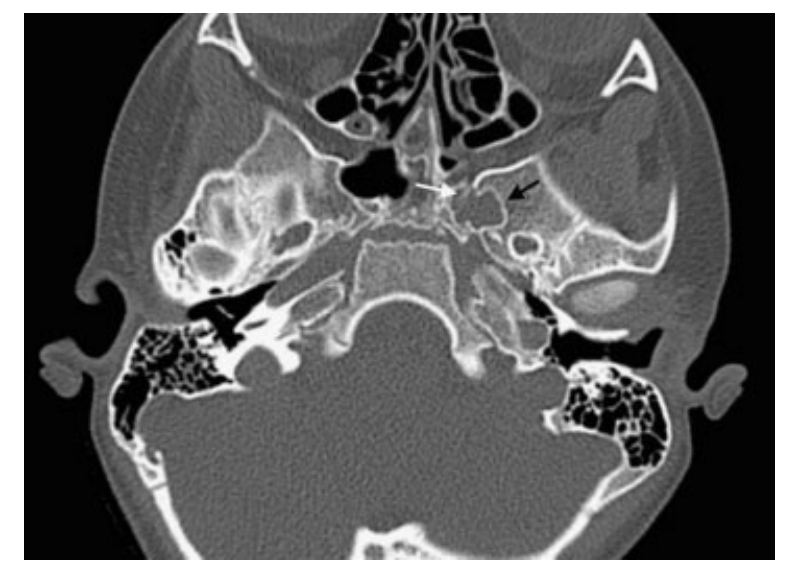

Fig. 4 Axial CT image showing a hypodense region of arrested pneumatization on the left side of the sphenoid bone, extending into the basisphenoid and pterygoid process. The lesion has a well-circumscribed sclerotic margin (black arrow), no mass effect, and nonexpansile characteristics with detectable curvilinear calcification (white arrow), with soft tissue and fat density. nostic imaging examination for various disease entities. While one case was being examined for reasons of left sixth nerve paralysis and headache, lesion identification on the sphenoid bone led to contrast-enhanced MR assessment but no enhancement was identified. During the patient's followup period, complaints and neurologic findings were seen to regress spontaneously. Thus, the symptoms were concluded to be unrelated to arrested pneumatization.

Radiologic imaging findings for arrested pneumatization were defined by Welker et al as well-circumscribed, sclerotic margins, with narrow transition zone, and curvilinear internal calcification lesions without mass effect and nonexpansile in CT examinations (-Fig. 4). MR imaging characteristics are in the form of hyperintense appearance, exhibiting fatty content on T1weighted images, no enhancement after gadolinium injection, and hyperintense appearance on T2-weighted images exhibiting microcytic content (-Fig. 3). In light of the literature, in the event of lesions satisfying the defined criteria, cases could be given the diagnosis of arrested pneumatization. These criteria include presence of lesions in expected pneumatization or known accessory regions, sclerotic margins, well-circumscribed, and nonexpansile, with fatty content and internal curvilinear calcification shown by CT and normal appearance in any of the related skull base foramina. ${ }^{3}$

In the differential diagnosis, fibrous dysplasia, ossifying fibroma, chondrosarcoma, osteomyelitis, chordoma, and bone metastases may be considered. However, in contrast to arrested pneumatization, those lesions do not exhibit fat content and generally have a mass effect on surrounding structures. ${ }^{3}$

\section{Conclusion}

Arrested pneumatization is also observable in the pediatric age group and displays similar imaging characteristics as in adults. Knowledge of the presence and imaging characteristics of this entity will prevent not only potential confusion in differential diagnosis by radiologists and clinicians, but also unnecessary advanced investigations and interventions.

\section{References}

1 Spaeth J, Krügelstein U, Schlöndorff G. The paranasal sinuses in CTimaging: development from birth to age 25. Int J Pediatr Otorhinolaryngol 1997;39(1):25-40

2 Scuderi AJ, Harnsberger HR, Boyer RS. Pneumatization of the paranasal sinuses: normal features of importance to the accurate interpretation of CT scans and MR images. AJR Am J Roentgenol 1993;160(5):1101-1104

3 Welker KM, DeLone DR, Lane JI, Gilbertson JR. Arrested pneumatization of the skull base: imaging characteristics. AJR Am J Roentgenol 2008;190(6):1691-1696

4 Kuntzler S, Jankowski R. Arrested pneumatization: witness of paranasal sinuses development? Eur Ann Otorhinolaryngol Head Neck Dis 2014;131(3):167-170

5 De Jong MD, Fassaert TA, Ranschaert ER. Arrested pneumatization of the skull base. JBR-BTR 2011;94(3):114-115

6 Jadhav AB, Tadinada A, Rengasamy K, Fellows D, Lurie AG. Clival lesion incidentally discovered on cone-beam computed tomography: A case report and review of the literature. Imaging Sci Dent 2014;44(2):165-169 